\title{
LA ENERGÍA COMO NÚCLEO EN EL DISEÑO CURRICULAR DE LA FÍSICA
}

\author{
PÉREZ-LANDAZÁBAL, M.C. ${ }^{2}$, FAVIERES, A. ${ }^{2}$, MANRIQUE, M.J. ${ }^{3}$ y VARELA, P. ${ }^{4}$ \\ ' I. Flectrónica de Comunicaciones (CSIC). Madrid. \\ 2 IB Mariana Pineda. Madrid. \\ ${ }^{3}$ IB Rey Pastor. Madrid. \\ IB Ramiro de Maeztu. Madrid.
}

\section{SUMMARY}

A research ${ }^{1}$ study on an approach to Physics teaching, at a basic level, centered on energy is presented. This study, framed on the Constructivist theory of tearning, has led to the design of curricula materials and their implementation and evaluation in secondary classrooms. An initial exploration of the prior beliefs of pupils about energy constituted the base of this curricula materials.

\section{INTRODUCCIÓN}

En este artículo se presenta un trabajo de investigación sobre la estructuración de la física, en un nivel básico, tomando como núcleo la energía. El estudio se ha llevado a cabo dentro del marco de la teoría constructivista de enseñanza-aprendizaje (Driver y Oldham 1986, Osborne y Freyberg 1985, Novak 1988) y se ha desarrollado en varias fases consistentes en:

- La exploración de las ideas alternativas de los alumnos referentes a la conceptuałización de la energía y sus cualidades;

- el diseño de materiales curriculares que tomen como base los esquemas conceptuales identificados en los alumnos de este nivel durante lá primera fase;

- la experimentación en el aula de estos materiales con una muestra total de 188 alumnos de $2^{\circ}$ de BUP pertenecientes a dos institutos de bachillerato del área urbana de Madrid. Esta experimentación se realizó durante los cursos 1989-90 y 1990-91;

- la evaluación de la eficacia didáctica de dichos materiales, entendida como una actividad en que la información recogida sirve para realimentar todo el proceso.

\section{EL PROBLEMA DE LA ENERGÍA EN LOS DISENOS CURRICULARES}

Existen diferentes criterios sobre la manera de abordar el tema, lo que ha dado origen a fuertes controversias:

Por un lado, la distinta conceptualización de la energía: hay autores (Warren 1982) que sostienen que se debe dar una definición operativa de la energía a partir del trabajo mecánico, mientras que otros (Duit 1981, 1987, López, Rupérez 1983, y una mayoría de los que han diseñado materiales curriculares sobre cl tema) defienden que se puede dar una definición descriptiva de la energía que posteriormente se irá completando.

En cuanto al punto de partida hay varios enfoques, como reseña Duit en un resumen sobre este tema (1986):

- Comenzar con el concepto de la energía como sustancia cuasimaterial (Schmid 1982). En esta línea pueden citarse los que inician el tema con transformaciones energéticas (por ejemplo, el Proyecto SCIS 1971), ya que utilizan, en la mayor parte de los casos, la imagen de flujo energético. Estos autores pertenecen a lo que Warren denomina grupo materialista.

- Partir de la conservación de la energía, centrándose en la importancia que los principios de conservación tienen 
en la física (Feynman et al. 1969). Este planteamiento presenta el inconveniente de requerir un gran esfuerzo de comprensión por parte de los alumnos más jóvenes.

Comenzar con el concepto de calor, por ser más fácil abordar experimentalmente la conservación de la energía a partir de esta idea (Proyecto COPES 1969).

Toda esta problemática surge del hecho innegable de que el enfoque tradicional en el estudio de la energía y de su conservación a partir de la noción de trabajo mecánico se ha mostrado poco efica $z$ en el aspecto de proporcionar una idea global de la energía, sus transferencias, trans. formaciones, conservación y degradación. Restringe el concepto de la energía al campo de la mecánica y no capacita a los estudiantes para aplicar el principio de conservación en situaciones variadas (Driver y Warrington 1985, Duit 1983), dejando también sin resolver el conflicto entre el principio de conservación y la degradación de la energía. Además, en el propio campo de la mecánica, no evita la confusión entre los conceptos de fuerza, trabajo, energía.

Consecuencia de todo esto es la opintón generalizada de que hay que modificar la enseñanza de la energía. En esta línea existen diversos grupos de trabajo e investigación que en los últimos años han elaborado cursos y proyectos curriculares que tratan el tema de la energía y su conservación desde un punto de vista distinto del tradicional en la enseñanza básica de la física, como el Proyecto CLIS de la Universidad de Leeds (1987), el «Learning Science Project» de la Universidad de Waikato (Stead 1980) y el proyecto del Seminario Axarquía (1989) en España.

\section{EXPLORACIÓN DE LAS IDEAS PREVIAS DE LOS ESTUDIANTES}

El primer paso de esta investigación ha consistido en una revisión bibliográfica sobre las ideas alternativas de los alumnos en este campo. Los trabajos existentes se pueden agrupar en dos grandes líneas: a) Conceptualización de la energía (Favieres et al. 1989, Kruger y Palacio 1992, Watts 1983), y b) Transferencia y conservación de la energía (Driver y Warrington 1985, Duit 19831986 , Favieres et al. 1989, Hierrezuelo y Molina 1990, Kruger y Palacio 1992, Solomon 1985).

De acuerdo con esta clasificación, en este trabajo se han utilizado pruebas de lápiz y papel del tipo asociación de palabras y elección múttiple que abarcan ambos campos (Anexo l). Para profundizar en las ideas subyacentes en las respuestas proporcionadas por Ios estudiantes, se realizaron entrevistas clínicas tipo Piaget a una muestra representativa de los mismos, cuyo objetivo era comprobar si correspondían a afirmaciones superficiales y fáciles de modificar o, por el contrario, respondián a estructuras más coherentes.
Conceptualización de la energía: prueba de asociación de palabras

Se ha elegido la modalidad de presentar una palabra estímulo -energía- y una lista de términos correspondientes a los conceptos con que usualmente la confunden los alumnos.

El análisis de las frases con que los alumnos justificaban su elección condujo a una clasificación de los significados que la palabra energía tiene para cllos. Las categorías establecidas se presentan en la tabla I, así como el número y porcentaje de alumnos asignados a cada categoría (dos asociaciones por cada uno de los 212 alumnos que realizaron esta prueba).

Tabla I

Asociación de palabras: Calegorías

\begin{tabular}{|l|c|c|}
\hline Categorias & Núm. alınnes & Porcentaji \\
\hline $\begin{array}{l}\text { I. Identificación con el } \\
\text { concepto de fuerza }\end{array}$ & 121 & 28,5 \\
\hline $\begin{array}{l}\text { II. Identificación con el } \\
\text { concepto de trabajo }\end{array}$ & 89 & 21,0 \\
\hline $\begin{array}{l}\text { III. Asociación exclusiva } \\
\text { energía con movimiento }\end{array}$ & 86 & 20,3 \\
\hline $\begin{array}{l}\text { IV. Energía como ingrediente } \\
\text { o depósio }\end{array}$ & 99 & 23,3 \\
\hline \begin{tabular}{l} 
V. Idea funcional de energía \\
\hline $\begin{array}{l}\text { VI. Concepción antropomórfica } \\
\text { de la energía }\end{array}$
\end{tabular} & 23 & 5,4 \\
\hline TOTAL & 424 & 1,4 \\
\hline
\end{tabular}

Estas categorías son similares a las descritas en la literatura. Sin embargo, cuantitativamente, la relación de la energía con la fuerza, el trabajo y el movimiento resulta mayor en nuestra muestra (un $70 \%$ de los estudiantes elige alguna de estas palabras entre las posibles). Una razón puede ser que, por su edad (15-16 años), los alumnos ya han visto algo de física en sus estudios primarios aunque no han alcanzado una discriminación clara entre los conceptos mencionados de fuerza, trabajo y energía.

En un segundo lugar aparece la idea de energía como ingrediente, asociada fundamentalmente con la pila eléctrica (14\%), pues tanto alimentos como explosivos no son elegidos prioritariamente. Por último, la idea antropomórfica de la energía es muy minoritaria, también en desacuerdo con los resultados de otros investigadores. 


\section{Transferencia y conservación de la energía: «camión de Micky» y «cambio imposible»}

El análisis de los resultados obtenidos en las diferentes pruebas aplicadas durante un estudio transversal previo (Favieres et al. 1989), en particular las explicaciones proporcionadas por los alumnos, nos condujo a seleccionar como pruebas definitivas para este trabajo dos de tipo general utilizadas por el proyecto CLIS (Brook y Driver 1984): «camión de Micky" y "cambio imposibles.

\section{Camión de Micky}

Su objetivo es explorar si los alumnos tienen asimilada la idea de energía acumulada (potencial) o si, por el contrario, asocian energía a movimiento (Solomon 1983, Watts 1983, etc.). También se pretende observar si introducen en su explicación los áspectos de conservación y degradación de la energía.

El análisis de los razonamientos con que los alumnos justifican su elección y de las entrevistas realizadas nos condujo a categorizar las respuestas tal como se presenta en la tabla II.

Tabla II

El camión de Micky: Categorías

\begin{tabular}{|l|c|c|}
\hline Cutcugorís & Núm. alumnos & Porcentaje \\
\hline $\begin{array}{l}\text { I. Interpretaciones aceptables } \\
\text { en términos de energía }\end{array}$ & 108 & 59 \\
\hline $\begin{array}{l}\text { II. Confusión } \\
\text { energia - fuerza }\end{array}$ & 7 & 4 \\
\hline $\begin{array}{l}\text { III. Identificación energía- } \\
\text { movimiento }\end{array}$ & 30 & 16 \\
\hline $\begin{array}{l}\text { IV. Aplicación incorrecta del } \\
\text { principio de conservación } \\
\text { de la cnergia }\end{array}$ & 11 & 6 \\
\hline \begin{tabular}{l} 
V. No codificables \\
\hline TOTAL
\end{tabular} & 28 & 15 \\
\hline
\end{tabular}

Hay que scñalar que la idea alternativa de energía asociada a movimiento se manifiesta tanto entre los alumnos que escogen la opción del camión en movimiento como entre aquéllos que, eligiendo la opción correcı, razonan de manera equivocada. Del análisis de las respuestas se deduce también que no reconocen la existencia de energía potencial.

No aparecen prácticamente alusiones a la degradación de la energía en las contestaciones. Sólo en un caso se cita el rozamiento, pero sin dar ninguna interpretación de tipo energético.

\section{Cambio imposible}

Su objetivo es comprobar si los alumnos son capaces de aplicar el principio de conservación, unido a la idea de degradación de la energía. La información que se puede obtener de esta cuestión es bastante amplia, lo que nos ha llevado a escogerla como prueba para evaluar el cambio conceptual experimentado por los alumnos después de la instrucción. Ia tabla IIl presenta la distribución de alumnos según los razonamientos empleados en sus respuestas.

Tabla III

Cambio imposible: Categorias.

\begin{tabular}{|l|c|c|}
\hline Caregorís & Núm. alumnos & Porcentaje \\
\hline $\begin{array}{l}\text { I. Interpretaciones aceptables } \\
\text { en términos de conservación } \\
\text { de la energía }\end{array}$ & 64 & 34,1 \\
\hline $\begin{array}{l}\text { II. Cambios intrínsecamente } \\
\text { imposibles }\end{array}$ & 47 & 25 \\
\hline $\begin{array}{l}\text { III. Fenómenos no relacionados } \\
\text { con la energía }\end{array}$ & 19 & 10,1 \\
\hline IV. Idea de rentabilidad & 17 & 9 \\
\hline V. No codificables & 41 & 21,8 \\
\hline TOTAL & 424 & 100 \\
\hline
\end{tabular}

Hay que destacar la existencia de cierta incapacidad para admitir la transformación de unas formas de energía en otras, como por ejemplo la transformación de energía química de un combustible en eléctrica en una central térmica o la de cnergía eléctrica en sonora en un altavoz. Tambiér se rechazan algunas transformaciones por criterios de rentabilidad (bombilla).

Como conclusión podemos afirmar que la muestra con que se ha trabajado no tiene asumido el principio de conservación de la energía conjuntamente con la idea de degradación. La idea de que en toda transformación real la energía total se mantiene constante, pero solamente es utilizable una energía menor que la suministrada no está en el bagaje científico de estos estudiantes.

\section{DESCRIPCIÓN DE LA EXPERIENCIA}

\section{Criterios básicos para el diseño de materiales}

En esta propuesta, se ha optado por dar una definición descriptiva: La energía se introduce como una magnitud fundamental, caracteristica de los sistemas, en virtud de la cual éstos pueden transformarse, modificando su estado o situación, asi como actuar sobre otros sistemas originando en ellos procesos de transformación (López 
Rupérez 1983). El trabajo y el calor se introducen con posterioridad como procesos de intercambio de energfa. En todo momento se intenta evitar la idea cuasimaterial de energía como flujo propuesta por Schmid y cuestionada por diversos autores.

La conceptualización elegida para la energía nos proporciona la base para el estudio posterior de la física y la química, centrado en las diferentes formas de energía (eléctrica, mecánica, térmica, etc.), su transferencia y su transformación. En todos los procesos estudiados se recalca la idea de conservación de la energía paralelamente a la idea de degradación: La energía se conserva pero, al ser utilizada, va perdiendo «calidad» y capacidad para ser empleada de nuevo (Varela et al. 1993).

Para dar una idea integrada de la ciencia, hemos destacado la importancia de la energía en la realización de procesos biológicos (nutrición, fotosíntesis, etc.) y tecnológicos (centrales eléctricas, electrodomésticos, etc.). Estas ideas quedan reflejadas en el esquema de la figura 1:

\section{Figura!}

Esquema del enfoque conceptual

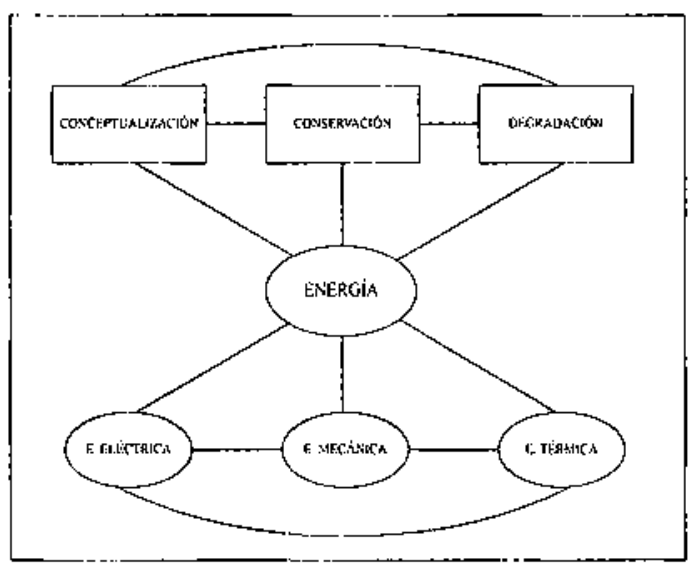

Este planteamiento está en la línea de la propuesta de Duit (1986) que dice que, independientemente del enfoque clegido, el estudio de la energía debe tener en cuenta cinco aspectos básicos: conceptualización, transferencia, transformación, conservación y degradación. Aunque estos cinco aspectos son de igual importancia, en la enseñanza se suele tratar menos el aspecto de la degradación.

Otro criterio empleado en el diseño de los materiales ha sido procurar que los alumnos se vean obligados a operar tanto en el dominio cotidiano como en el dominio científico ya que, como nos indica Solomon (1983), los alumnos que han demostrado su capacidad para pasar de un dominio a otro sin errores poseen una comprensión más firme de la abstracción que supone la energia $y$ de sus transformaciones.

\section{Descripción de las actividades didácticas}

El materia! de trabajo consiste en seis actividades que comentamos a continuación.

\section{Actividad 1. Ideas previas sobre energía}

Esta actividad comienza con la exploración de las ideas previas de los alumnos sobre la conceptualización de la energía. A partir del análisis de los resultados obtenidos en el aula -discusión en grupos de una selección de las frases proporcionadas en la prueba de asociación de palabras-, se hace patente la confusión entre los términos energía, fuerza, trabajo, etc., provocando el conflicto cognitivo al contrastar el alumno sus ideas con las de sus compañeros y las aceptadas por la ciencia actual.

\section{Actividad 2. Necesidad de la energra}

Los alumnos reflexionan sobre la necesidad de la energía, trabajando en dos campos distintos:

a) energia para mantenernos vivos;

b) energía para mejorar nuestra vida cotidiana.

\section{Actividad 3. Cuantificación de la energía}

Los alumnos anatizan y cuantifican el consumo energético, manejando tablas de datos, transformando unidades e interpretando gráficos. El trabajo se rcaliza en las dos vertientes tratadas en el punto anterior:

a) Energía para mantenernos vivos: A partir de tablas del gasto calórico correspondiente a distintas actividades y tablas del contenido calórico de alimentos, los alumnos elaboran una dieta. Como consecuencia y ampliación del trabajo se tratan aspectos bioquímicos de la digestión.

b) Energía para mejorar nuestra vida cotidiana: Se determina el consumo de energía eléctrica de diversos electrodomésticos, a partir de los datos de potencia y tiempo de utilización. Esto da pie para tratar cuestiones relacionadas con el entorno familiar como los recibos de compañias eléctricas, del gas, etc.

\section{Actividad 4. La energía y sus transformaciones}

A partir del estudio de las transformaciones de la energía, se plantea el problema del suministro energético, manejando términos del lenguaje común como fuentes de energía, energías renovables, energía limpia, ahorro energético, etc., tratando de integrar los dos dominios de conocimiento, científico y cotidiano, anteriormente citados.

También se estudian aquí los distintos tipos de centrales eléctricas, lo que permite abordar de manera cualitativa los fenómenos de inducción electromagnética. Los atumnos 
realizan un trabajo monográfico sobre la obtención de energía cléctrica, destacando la posible utilización para ello de energías renovables.

\section{Actividad 5. La energía y su conservación. Energía útil}

En esta actividad, el trabajo de los alumnos se desarrolla en dos líneas complementarias: a) la conservación de la energía en cualquier tipo de transformación, y $b$ ) la imposibilidad de convertir toda la energía inicial en energía utilizable.

La primera tarea consiste en dibujar diagramas cuantitativos de diferentes transformaciones, indicando todos los tipos de energía que aparecen en ellas. Se insiste en que el diagrama debe visualizar claramente que la suma de las energias finales tiene que ser igual a la suma de las energías iniciales. En la figura 2 se presenta el modelo que, tras diversos ensayos, se consideró mas conveniente para la comprensión del principio de conservación.

Figura 2

Ficha de trabajo sobre las translormaciones energéticas.

\section{LAS TRANSFORMACIONES ENERGÉTICAS}

Vamos a intentar representar gráficamente de un modo cuantitativo un cjemplo de transformación energética.

Una manera de hacerlo puede ser ésta:

Encrg̣iat susuinixtroda E.ncrgéius transtortnudih

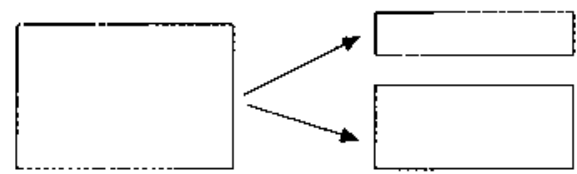

Los rectángulos indican las diterentes clases de cnergía que intervicnen en el proceso representado.

Utiliza esta representación para esquematizar las transformacio nes energéticas en cl caso de una batidora cléctrica e indica qué cłase de cnergía representa cada rectángulo.

Utilizando estos diagramas en casos sencillos, como la batidora eléctrica, se puede deducir que, de todas las formas de energía que aparecen al final del proceso, solamente una de ellas va a utilizarse para el objetivo deseado. Esta idea de energía útil sirve para introducir el concepto de rendimiento de la transformación, que sc aplicará a distintos aparatos de uso común (motor del coche, calentador eléctrico, etc.).

La actividad termina planteando la pregunta ¿Qué suce de con la energía que no se utiliza?, la cual da paso a la introducción de la degradación de la energía.

\section{Actividad 6. Degradación de la energía}

Los alumnos analizan cuantitativamente las transformaciones energéticas que tienen lugar en una central eléc- trica. A partir del hecho de que, en cada una de las etapas, una parte de la energía se transforma en energía térmica, «no aprovechable», se puede llegar al concepto de degradación de la energía. Así los alumnos asumen que, en toda transformación, la energía se conserva pero pierde calidad, se degrada, ya que acaba transmitiénclose al ambiente de donde no es posible recuperarla.

Este concepto se refuerza analizando otros casos; por ejemplo, las transformaciones de energia que ocurren ciando un niño que va en bicicleta se para o cuando una piedra que cae llega al suelo.

Como aspectos complementarios se ha tratado el aislarniento térmico de las viviendas, la contaminación sonora y el efecto invernadero.

\section{Metodología del trabajo en el aula}

La metodología utilizada en el aula tiene que estar de acuerdo con el modelo constructivista de enseñanzaaprendizaje con el cual se ha trabajado en esta investigación, tal como se indica en la introducción. En esta línea, se pretende que los alumnos:

- pongan en cuestión sus ideas;

- discutan estas ideas con sus compañeros;

- emitan hipótesis acerca del comportamiento de determinados sistemas;

- contrasten sus hipótesis con los resultados obtenidos en la experimentación o aportados, en su caso, por el profesor;

- apliquen las nuevas ideas a otras situaciones.

Los alumnos trabajaron en las actividades que se les propuso en grupos de cuatro, exponiendo y discutiendo sus opiniones, las cuales posteriormente se ponían en común con el resto de los equipos para obtener una conclusión general. También se realizaron actividades individuales, como redacciones o trabajos monográficos que quedan reflejados en el cuaderno de clase de cada alumno.

La experiencia se ha realizado siempre al comienzo de la asignatura dado su carácter de núcleo básico para el estudio posterior de física y química. La duración del trabajo en el aula ha sido de 20 horas, lo que corresponde aproximadamente a cinco semanas.

\section{EVALUACIÓN}

El modelo de evaluación utilizado responde a una aproximación metodológica a la investigación-acción, que se caracteriza por una práctica social reflexiva, donde no se distingue entre la práctica que se investiga y el proceso 
de investigación de esa práctica (Lewin 1946, Porlan 1987). En consecuencia, se ha realizado una evaluación en que la información recogida el primer año ha servido para modificar tanto los materiales diseñados como su aplicación dentro del aula.

Este tipo de evaluación implica trabajar en dos campos complementarios, el campo de los alumnos y el del proceso de enseñanza-aprendizaje.

En el primer campo ha sido preciso medir de manera cuantitativa el cambio conceptual producido en los alumnos, asf́ como evaluar su persistencia a lo largo del tiempo. También se han planteado pruebas, más o menos convencionales, para contrastar el avance experimentado, tanto en conteridos como en destrezas.

En la evaluación del proceso se trata de juzgar la validez del currículo propuesto y de la metodología empleada, así como determinar las principales dificultades que se presentan en el proceso de cambio conceptual. Para conseguir el mayor nivel posible de fiabilidad en nuestras apreciaciones se han utilizado diversos procedimientos: encuesta actitudinal, entrevistas, cuadernos de trabajo, grabaciones en audio y vídeo del trabajo en el aula, etc.

\section{Evaluación del cambio conceptual}

Se utilizó la técnica del pretest/postest, eligiendo para ello la prueba de Cambio imposible (Brook y Driver 1984) descrita anteriormente. Después de realizado el postest se procedió a entrevistar a un grupo de seis alumnos con el fin de profundizar en los razonamientos que habían utilizado en sus contestaciones. En el anexo II se presenta un resumen de dichas entrevistas. Los datos obtenidos se presentan en la siguiente tabla.

Tabla IV

Evaluatción del cambio conceptual.

\begin{tabular}{|l|c|c|}
\hline Cacgorias & Núm. alumnos & Porcentaje \\
\hline Evolucionan hacia ideas & 85 & 45,2 \\
\hline $\begin{array}{l}\text { Inicialmente presentaban } \\
\text { ideas científicas }\end{array}$ & 64 & 34,1 \\
\hline $\begin{array}{l}\text { Persisten en ideas } \\
\text { abtcrnativas }\end{array}$ & 33 & 17,5 \\
\hline Respuestas no codificables & 6 & 3,2 \\
\hline TOTAL & 188 & 100 \\
\hline
\end{tabular}

El análisis de los reșultados nos permite afirmar que el $45 \%$ de los alumnos ha experimentado un cambio con- ceptual positivo. Este dato, unido al $34 \%$ que ya inicialmente respondía correctamente, nos da un $79 \%$ de estudiantes capaces de detectar el cambio que no se puede producir, explicándolo de forma razonada

\section{Evaluación del nivel de conocimientos}

Además de la prueba Cambio imposible, se plantearon preguntas abiertas, en las cuales los alumnos tenían que dibujar un diagrama cuantitativo de las transformaciones energéticas de un determinado proceso y calcular, a partir de su diagrama, el rendimiento del mismo. Los resultados se presentan en la tabla $\mathrm{V}$.

Tabla V

Evaluación del cambio conceptual.

\begin{tabular}{|l|c|c|}
\hline Respuestas correctas & Núm. alumnos & Porcentaje \\
\hline Diagrama cuantitativo & 125 & 66,5 \\
\hline $\begin{array}{l}\text { Rendimiento de la } \\
\text { transformación }\end{array}$ & 117 & 62,2 \\
\hline
\end{tabular}

Se puede observar que, aproximadamente, las dos terceras partes de los alumnos resolvieron satisfactoriamente toda la tarea. Si comparamos estos resultados con los obtenidos usualmente en nuestros exámenes, podemos considerarlos positivos.

Las respuestas a la prueba utilizada como postest fueron cuantificadas entre 0 y 10 puntos con el fin de poder adjudicar una puntuación numérica a cada alumno. Los resultados globales son: sobre una muestra de 188 alumnos, 130 de ellos obtuvieron una calificación superior a 5 , lo que nos da un $69 \%$ de «aprobados», resultado, que como los obtenidos anteriormente, nos resulta alentador.

Al cabo de nueve meses de terminada la experiencia se aplicaron las mismas pruebas para medir la persistencia del cambio concepiual. Los resultados fueron altamente satisfactorios, aunque no se recogen en este trabajo por considerar que la muestra estaba sesgada en sentido positivo debido a las dificultades de seguimiento de aquellos alumnos que no optaron por física y química en $3^{\circ}$ de BUP.

\section{Evaluación del proceso}

Observación sistemática del trabajo realizado por los alumnos y el profesor. Grabaciones

Este tipo de observación a través de un agente externo -en este caso otros miembros del grupo- proporciona datos más objetivos e imparciales para la evaluación. Se construyó un mapa del aula con la situación de los alumnos, centrándose la observación en una serie de puntos tales como: atención, actitud en la clase, trabajo 
Tabla VI

Prueba actitudinal.

I. ¿il aprendizaje de todos los contenidos correspondientes a la unidad de energía te ha resultado...?

2. ¿La metodología empleada, comparada con la que normalmentc se utiliza en las clases, te ha parecido...?

3. ¿El tipo de materiales didácticos con que hemos trabajado, en contraposición con el hibro de texto te ha faciliado el aprendizaje?

4. ¿El trabajo en grupo ha favorecido tu aprendizaje?

5. ¿El tipo de aclividades que hemos desarrollado tc ayudará en el futuro a resolver y comprender situaciones dc tu vida colidiana?
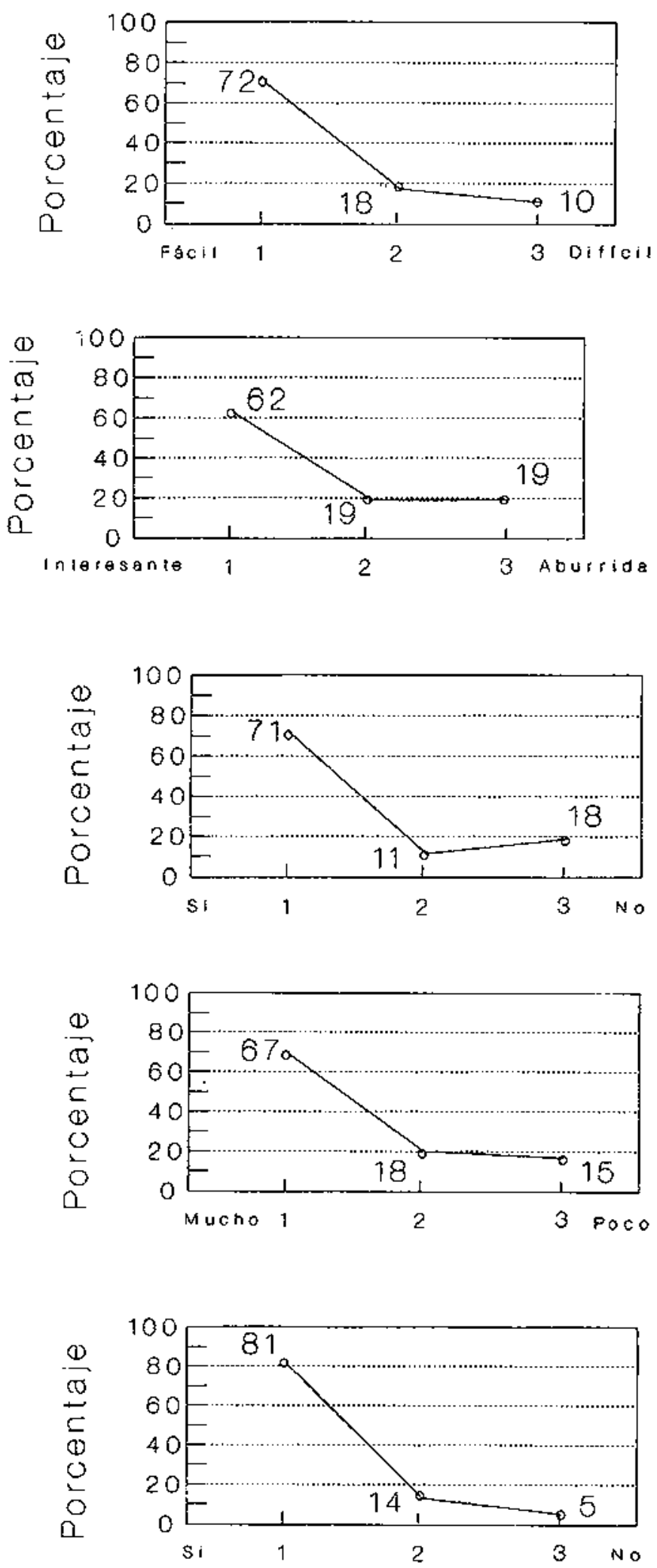
en los equipos, comprensión de los materiales y duración de cada actividad. También se observó el papel que el profesor desempeña en el desarrollo de las actividades.

Paralelamente se recogieron en cintas de audio las discusiones de los equipos, puestas en común, ctc. y se grabó en vídeo el desarrollo de algunas de las clases.

El análisis posterior de todos los datos recogidos puso de manifiesto que el grado de participación e interés por parte de los alumnos es más elevado que el observado en «situaciones más estándar».

\section{Cuadernos de clase}

Constituye uno de los instrumentos mas valiosos de la evaluación, ya que el alumno recoge en él su trabajo diario: material utilizado en clase, actividades desarroliadas y opiniones del grupo sobre dichas actividades, puestas en común y conclusiones de toda la clase, así como todas las actividades que se realizaban en casa.

\section{Prueba actitudinal}

Para completar la información obtenida con la evaluación externa se realizó una prueba de lápiz y papel, ampliada con entrevistas a una pequeña muestra de alumnos. Los resultados obtenidos, tabulados en tanto por ciento, sc presentan en la tabla Vl de forma gráfica y confirman nuestra hipótesis inicial de que unos materiales y una aplicación en el aula que cumpliera los requisitos descritos tendrían una respuesta positiva por parte de los alumnos. El porcentaje de éstos que se encuentra on el cxtremo más positivo del diferencial semántico aplicado para cada una de las preguntas, oscila entre un $62 \%$ (metodología del trabajo en clase) y un $81 \%$ (implicaciones que tiene lo aprendido en el aula en las vivencias de los estudiantes fuera de ella). Estos valores son muy superiores a los obtenidos en pruebas similares realizadas con alumnos inmersos en modelos de enseñanza expositivos. El extremo más negativo de los diferenciales planteados no es escogido nunca por más del $19 \%$ de los encuestados $\mathrm{y}$, del análisis de las contestaciones vertidas en las entrevistas, parece deducirse que estos alumnos prefieren utilizar los materiales didácticos como aplicaciones prácticas del tema tratado en forma tradicional.

\section{CONCLUSIONES}

Está ampliamente aceptado que los conocimientos científicos que construyen los alumnos tienen que tener una estructura coherente y global donde puedan articularse los conceptos que van adquiriendo. En este sentido, y como ha quedado expuesto a lo largo del artículo, hemos utilizado el concepto de energía como hilo conductor para el desarrollo de todas las actividades. Sus atributos, posibilidad de transferencia y transformación, conservación y degradación en todos los procesos reales han sido puestos de manifiesto en los diferentes contextos trabajados -nutrición, fotosíntesis, cuerpos en movi- miento, electrodomésticos, centrales eléctricas, etc.--, dando a los alumnos suficientes oportunidades para conseguir un aprendizaje significativo en el sentido definido por Ausubel (1978).

Por otra parte, el tratamiento al mismo nivel del principio de conservación de la energía y del concepto de degradación ha supuesto, en nuestra opinión, una comprensión más acorde con la intepretación científica del citado principio.

Los resultados de este trabajo podrían ser una aportación interesante a las numerosas investigaciones realizadas sobre la existencia de esquemas conceptuales alternativos en tos estudiantes así como sobre las posibles estrategias a utilizar para conseguir su evolución, plasmándolas en unos materiales que, además de cumplir los requisitos de significatividad lógica y psicológica, han sido suficientemente contrastados.

En cuanto a la metodología de la investigación, enmarcada en la línea de investigación-acción, ha consistido en una espiral de actividades que supone un diagnóstico de la sifuación problemática, la formulación de estrategias de acción, la puesta en práctica y evaluación de dichas estrategias y una nueva diagnosis del estado actual de la situación.

Como se desprende de los datos aportados en la evaluación, un enfoque de estas características nos ha permitido contemplar el proceso desde ángulos muy distintos y llegar a las conclusiones siguientes:

\section{Cambio conceptual experimentado por los alumnos}

Respecto a los conocimientos adquiridos por los alumnos, los resultados de las pruebas demuestran que una mayoría de ellos es capaz de modificar sus ideas adoptando las científicas y, lo que es más importante, persiste en ese cambio conceptual. En nuestra opinión éste es un punto clave del resultado de la enseñanza, por la dificultad que encicrra modificar de forma estabie los esquemas conceptuales previos de los alumnos.

Por otra parte, el nivel de conocimientos adquiridos es aceptable, sobre todo si se considera la dificultad inherente al propio tema y a que el principio de conservación de la energía no se ha restringido a procesos dentro del campo de la mecánica, sino que se ha aplicado a una gran variedad de transformaciones, en las que se cuantifica esta energía.

Es importante resaitar que el tratamiento del tema ha permitido dotar de significado físico expresiones del mundo cotidiano, posibilitando a los alumnos integrar los ámbitos científico y familiar.

\section{Una enseñanza para mejorar las actitudes}

La prueba actitudinal refleja que los alumnos cstán satisfechos, en general, con la metodología empleada y consideran que lo aprendido tiene una aplicación directa en su vida cotidiana, cuestión esta que no es muy fre- 
cuente entre los estudiantes de física en sus primeros niveles. Los valores obtenidos son muy superiores a los obtenidos en pruebas similares realizadas con alumnos inmersos en modelos de enseñanza expositiva.

Como comentario final queremos indicar que los materiales diseñados y la metodología de trabajo en cl aula no parecen ser suficientes para conseguir el cambio conceptual deseado en la totalidad de los alumnos. Este hecho pone de manifiesto la extraordinaria complejidad del proceso de aprendizaje así como la diversidad de origen de las variables que intervienen y la necesidad de un análisis detallado de las causas por las cuales aparecen conceptos tan difícilmente modificables.

\section{REFERENCIAS BIBLIOGRÁFICAS}

AUSUBEI, P.D., 1978. Psicología educativa. Un punto de vista cognoscitivo. (Trillas: México).

BROOK, A y DRIVER, R, 1984. Aspects of Secondary students" Understanding of Energy. Children's Learning in Science Research Group. (CSSME: University of Leeds).

DRIVER, R. y OLDHAM,V., 1986. A Constructivist Approach to Curriculum Development in Science, Studies in Science Education, 13, pp. 105-122.

DRIVER, R. y WARRINGTON, L., 1985. Students' use of the principle of energy conservation in problem situations, Physics Education, 20, pp.171-176.

DUIT, R.,1981. Understanding energy as a conserved quantity. Remarks in the article by R.V. SexI, European Journal of Science Fiducation, 3 (3), pp. 29 -301

DUIT, R.,1983. Energy conceptions held by studients and consequences for Science teaching. Proceedings of an International Seminar on misconceptions in Science and Mathematics. Ithaca, Cornel] University 1983.

DUIT, R., I986. In search of an energy concept, en Driver, R. y Millar, R. (eds.), Energy matters, pp. 67-101. Centre for Studies in Science and Mathematics Education, University of Leeds.

DUIT, R., 1987. Should energy be illustrated as something quasi-matcrial?, International Journal of Science Education, 9 (2), pp. I39-145.

FAVIERES, A., MANRIQUE, M.J. y VARELA, P., 1989. Una aplicación del modelo constructivista al currículo de Física en Bachillerato. Proyecto subvencionado por la Consejería de Educación de la Comunidad Autónoma de Madrid y por la Dirección General de Renovación pedagógica del MEC.

FEYNMAN, R.P., LEIGHTON, R.B. y SANDS, M., 1969. The Feynman lectures on Physics. (Reading/Mass.: AddisonWesley).

HIERREZUELO, J. y MOLINA, E, 1990. Una propuesta para la introducción del concepto de energía en el bachillerato, Enseñanza de las Ciencias, 8 (1), pp. 23-30.

KRUGER, C., PALACIO, D. y SUMMERS, M., 1992. Survey of English Primary Teachers' Conceptions of Force, Energy, and Materials, Science Education, 76 (4), pp. 339-351.

\section{RECONOCIMIENTOS}

Los dibujos del anexo I han sido tomados de los estudios de Brook y Driver (1984). Deseamos agradecer a Angela Brook y a Rosalind Driver su permiso para usar las pruebas y dibujos "camión de Micky" y «cambio imposible» en nuestro estudio.

\section{NOTA}

' Eșa investigación ha sido subvencionada por el CIDE.

LEWIN, K., 1946. Action - Research as Minority Problems, Journal of Social /ssues, 2, pp. 34-46.

I.ÓFEZ RUPEREZ, F. et al., 1983. Las nociones de trabajo y energía. Análisis conceptual y didáctico, Bordón, 249, pp 497-506.

NOVAK, I.D., 1988. Constructivismo humano: un consenso emergente, Enseñanza de las Ciencias, 6 (3), pp. 213-223.

OSEORNE, I.R. y FREYBERG, P., 1985. Learning in Science. The implications of children's Science. (Heinemann: L ondres)

PORLAN, R., 1987. El maestro como investigador en el aula Investigar para conocer, conocer para enseñar, Investigación en la escuela, 1, pp. 63-70.

PROYECTO CLIS, 1987. Children's Learning in Science Froject, Secondary Students' ideas about energy. I.eeds University.

PROYECTO COPES, 1969. Conceptually Oriented Program in Elementary School. Citado por Duit, 1986.

PROYECTO SCIS, 1971. Science Curriculum Improvement Situdy. Citado por Duit, 1986.

SCHMID, B.G., 1982. Energy and its carriers, Physics Education, 17 (5), pp. 212-218.

SEMINARIO AXARQUIA, 1989. Aprendizaje de Fisica y Química. (Editorial Ezelvir: Vélez-Málaga).

SOLOMON, J., 1983. Learning about energy: how pupils think in two domains, European Journal of Science Education, 5 (1), pp. $49-59$.

SOLOMON, J., 1985. Teaching the conservation of energy, Physics Education. 20, pp. 165-170.

STEAD, B., 1980. Learning in science project: Energy. Working Paper n. 17. (University of Waikato: Hamilton, N.Z.).

VARELA, P.,FAVIERES, A., MANRIQUE, M.I. y PEREZ LANDAZÁBAL, M.C., 1993. Iniciación a la Fisica en el marco de la teoría constructivista. (Centro de Publicaciones del MEC: Madrid).

WARREN, J.W., 1982. The nature of energy, European Journal of Science Education. 4 (3), pp. 295-297.

WATTS, D.M., 1983. Some alternative views of energy, Physics Education, 18, pp. 213-217. 
ANEXO I

PRESENTACIÓN DE LAS PRUEBAS

\section{ASOCIACIÓN DE PALABRAS}

1. Entre las palabras que se indican a continuación, elige DOS, las que te parezcan más relacionadas con la energía.

$\begin{array}{ll}\text { Alimentos } & \text { Movimiento } \\ \text { Electrodomésticos } & \text { Atleta } \\ \text { Explosivos } & \text { Pila eléctrica } \\ \text { Fuerza } & \text { Trabajo }\end{array}$

2. Escribre dos frases que indiquen la relación entre la energía y cada una de las palabras que has elegido.

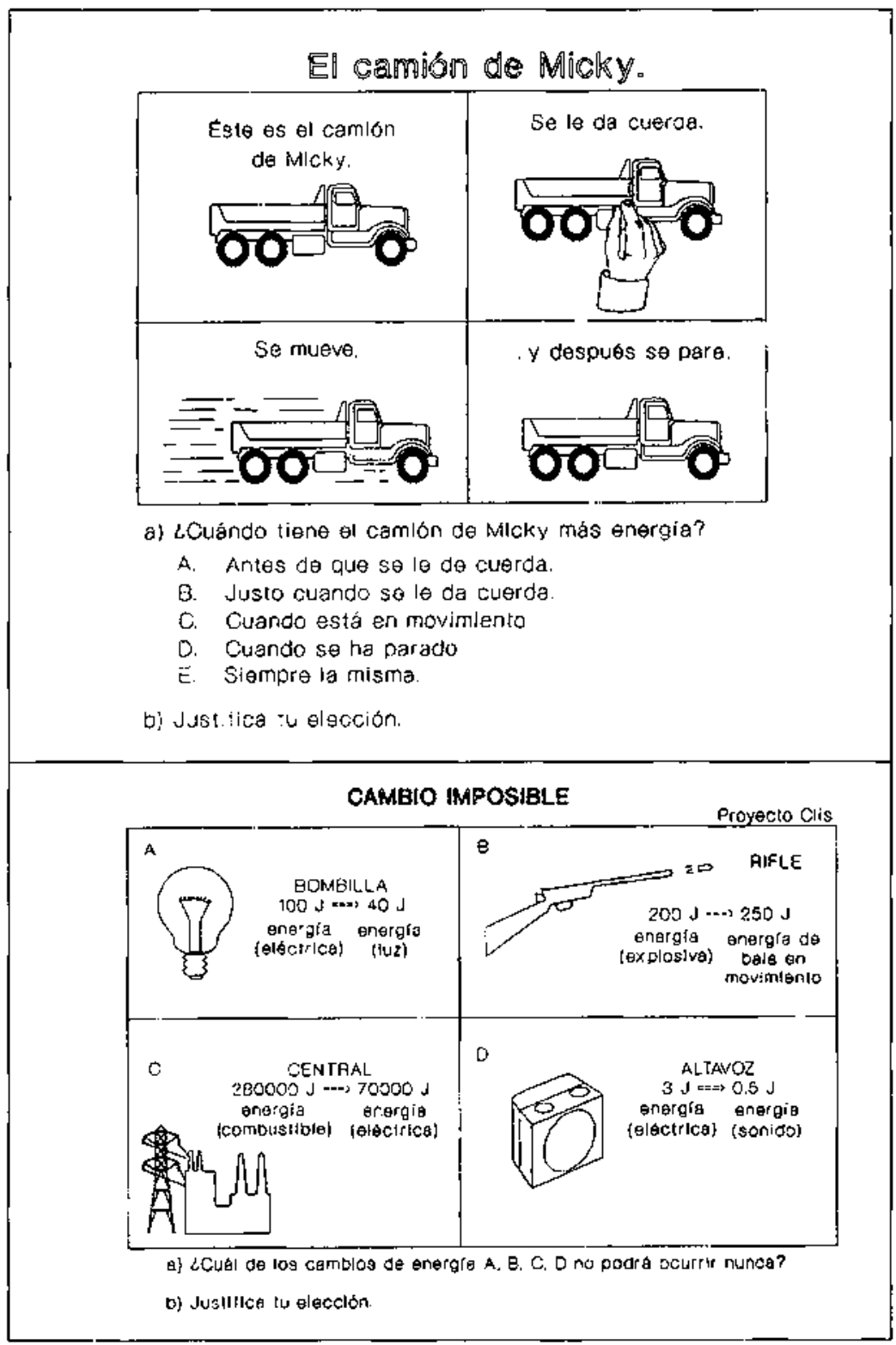




\section{ANEXO II \\ RESUMEN DE LAS ENTREVISTAS REALIZADAS AL, FINAL DEL PROCESO DE ENSEÑANZA}

Las respuestas corresponden a dos categorías: alumnos que han asumido la idea científica y alumnos que persisten en una idca alternativa.

a) Estudiantes que consideran el rifle como «cambio imposible» y razonan sobre ello:

\section{Alumno 1}

Profesora: -En el pretest dijiste que el cambio imposible era el del altavoz porque el sonido no era una energia. ¿Qué te ha hecho cambiar de opinión?

Alumna: -Que en una transformación es imposible que la energía que tenga el cuerpo al que se le ha transmitido sea mayor que la que tú transmites, que le das a ese cuerpo.

P: -¿Podrias explicarlo un poco mejor?

A: -Pues que si un cuerpo tiene una determinada energía ¿cómo va a trasmitir a otro cuerpo más energía de la que tiene?

\section{Alumno 2}

P: -¿Podrias explicar por qué has escogido el rifle?

A: -Sí, porque en los otros tres casos, de un paso de energia a otro se pierde energí, en el del rifle es el ínico en el que se gana, y yo creo que no se puede ganar de un paso de transformación energética a otra porque no sólo la energía es de la bala, también hay energia sonora y térmica...

\section{Alumno 3}

P: - ¿Puedes justificar tu elección?

A: - Pues yo he cogido el $B$, que es el del rifle que tiene una fuerza de 200 julios, o sea una energia, y la bala sale a $250 \ldots$ entonces yo creo que eso es imposible porque en el cambio de energia que se le da a la bala no puede poseer más fuerza de la que posee el rifle si la bala es disparada por ese rifle. (Hay una confusión fuerza-energía.)

\section{Alumno 4}

P: - ¿Te gustaría dar una explicación más amplia de por qué escogiste el caso B?

A: -Si un objeto tiene una energía almacenada no puede gastar más de esa energía, al moverse, al realizar un cambio, sino que utiliza esa energía y, al revés, siempre pierde algo y la energia se degrada. (Introduce la expresión energía degradada.)

b) Estudiantes que consideran la central térmica como «cambio imposible», ya que no tienen una tỏea clara de cómo se puede obtener energía eléctrica a partir de combustibles:

\section{Alumno 5}

P: ¿¿A qué te refieres con la "energía térmica en la central»?

A: -A la energía de la combustión del carbón, que para transformarla en eléctrica se necesita un aparato.

P: - ¿Tienes ideas del aparato?

A: -No. Yo sabía que en la central hidráulica la mecánica se convertía en eléctrica por un generador pero en este caso no.

\section{Alumno 6}

P: - ¿Por qué no obtienes energía eléctrica en el caso $C$ ? ¿Qué te faltu?

A: -Pues los medios para sacarla.

P: -¿Y tienes una idea de qué medios son ésos?

A: -Con el carbón no lo sé.

P: $-i Y$ con otro si?

A: -Si, por ejemplo... el agua que hay en una presa [... J se abren las compuertas y el agua cae a velocidad y abajo hay como una especie de rueda, o sea con turbinas, y al caer el agua desde cierta altura hace mover las turbinas que a su vez hace mover un generador y éste produce la energía eléctrica. 